\title{
Comparative Study between Breast Reduction Surgery in Macromastia With and Without Ultrasonographic Pedicle Determination
}

\author{
HAMZA F., M.D.; ABOUL FOTOUH A., M.D.; EL-BANOUBY T., M.D.; AWADEEN A., M.D. and \\ SHARAF M., M.Sc. \\ The Departments of Plastic Surgery \& Burn and Radiodiagnosis, Al-Azhar University, Cairo, Egypt
}

\begin{abstract}
Background: When performing breast reduction surgery, it is very important to preserve the vascularity of tissues, especially of the nipple-areolar complex (NAC). Preoperative ultrasonographic color Doppler imaging is used for determination of major supplier vessels of the nipple-areola complex. Pedicles containing these vessels were designed for reduction mammaplasty.

Patient and Methods: The study was done at Al-Azhar University Hospitals from April 2014 and April 2016 including 24 cases ( 48 breasts) with bilateral macromastia and age group 20-48 years with follow-up period 6 month. Group (A) 12 cases submitted for breast reduction using preoperative ultrasonographic color Doppler imaging for pedicle determination, group (B) 12 cases submitted for breast reduction via inferior pedicle technique.
\end{abstract} group.

Result: The selected cases were operated according their

In Group A: 12 cases after preoperative Doppler ultrasonography for each breast, the pedicle determined was; six cases with inferior pedicle design, four cases with superomedial pedicle design, and two cases with superior pedicle design, none of the patients had areola necrosis.

In Group B: All cases underwent reduction mammoplasty using inferior pedicle technique, none of the patients had areola necrosis.

Conclusion: Preoperative ultrasonographic color Doppler imaging for evaluation of the main nipple-areola complex vascular supply has certain advantages. Therefore, we strongly advise using this modality for evaluation of the main nippleareola complex vascular supply that specify which type reduction mammaplasty techniques for every patient.

Key Words: Breast vascularity - Macromastia - Ultrasonographic color Doppler-Pedicle determination.

\section{INTRODUCTION}

Breasts are regarded as the expression of feminity and sexual appeal. Breast reduction surgery provides physical comfort and improved life quality which was diminished by Macromastia. Thus hav- ing aesthetically pleasing breasts are very important especially for young women [1].

Numerous methods over the years have been designed for breast reduction to reduce their size and weight. The goals of the patient suffering from large breasts are as follows: To have relief of their physical symptoms; to have an improved selfimage with regard to the shape, size, harmony, and symmetry of their breasts, and, if possible, preserve sensation and function; for this to be performed in a safe manner and to not require additional surgery; and to have the best/least scars possible [2].

In the very large breast, many plastic surgeons are concerned about using pedicle techniques, especially in extremely large breasts (e.g., $>40 \mathrm{~cm}$ pedicle length), out of fear of NAC necrosis. Hence, some surgeons still use the amputation/free nipple graft technique, although it has distinct disadvantages, including loss of sensation, poor nipple projection, and loss of lactation [3].

\section{Important neurovascular anatomy:}

Embryologically, the breast develops as a fourth intercostal space structure, and it has an artery and venae comitantes that come up generally between the fourth and fifth ribs, originating in the internal thoracic vascular system. The superficial blood supply comes mainly from the internal thoracic artery as well, with contributions from the superficial branch of the lateral thoracic system and some contribution from the thoracoacromial system. As the breast develops during puberty, the superficial skin and subcutaneous tissue through which the vessels travel gets pushed outward. These vessels course deeply around the periphery of the breast but travel up and around the breast parenchyma toward the nipple [4]. 
A study by van Deventer in 2004 specifically investigated the blood supply to the NAC using cadaveric dissections of 27 adult female breasts and made a number of important observations. This study found a large variation in the pattern of blood supply to the NAC. None of the dissected breasts had identical vascular patterns, even when the two breasts of the same cadaver were compared. Therefore, it was concluded that it is impossible for the surgeon to predict the specific vascular pattern to the NAC [5].

A further important finding by van Deventer in 2008 was that in all the dissected breasts, the NAC received one or more perforating arteries from the upper four perforating branches of the internal thoracic artery. A designed pedicle can be regarded as an arterialized flap provided all four upper perforators are included in the pedicle. However, if all four upper perforators are not included in the pedicle, there may be a risk that the pedicle is a random flap [5].

\section{PATIENTS AND METHODS}

This was a prospective study carried out between April 2014 and April 2016. Including Twenty four (24) females patients (48 breasts) were recruited to the study presented with bilateral macromastia were selected and submitted for reduction mammaplasty in Al-Azhar University Hospitals, and were categorized in random fashion into 2 groups.

Each group containing Twelve (12) cases (24 breasts):

Group (A) submitted for breast reduction using preoperative ultrasonographic color Doppler imaging for pedicle determination.

Group (B) submitted for breast reduction via inferior pedicle technique without using preoperative ultrasonographic color Doppler imaging.

The mean age of 33.6 years (range, 20 to 43 years) were included in the study. Patients were followed-up for 6 months (range, 6 to 9 months) on average. Mean body mass index value was (31.59) in group A and (32.67) in group B 32.6 $\mathrm{kg} / \mathrm{m}^{2}$ (range, 25 to $35 \mathrm{~kg} / \mathrm{m}^{2}$ ). Patient data were recorded, including age, weight, body mass index, additional diseases. Standard measurements, such as suprasternal notch-to-nipple were recorded (Table 1).

Preoperative ultrasonographic pedicle determination:

Patients were analyzed by the same ultrasonographist before surgery and marked in supine posi- tion with $12-$ to $18-\mathrm{MHz}$ linear probe $\left(\right.$ Aplio $^{\mathrm{TM}}$ 400; Toshiba, Japan) Doppler ultrasonography. The probe was placed on the breast starting around the areola and traveling medially, laterally, caudally, and cranially. Vessels in continuity with the areola were marked.

Table (1): Patient demographics, breast measurements, and resection weights.

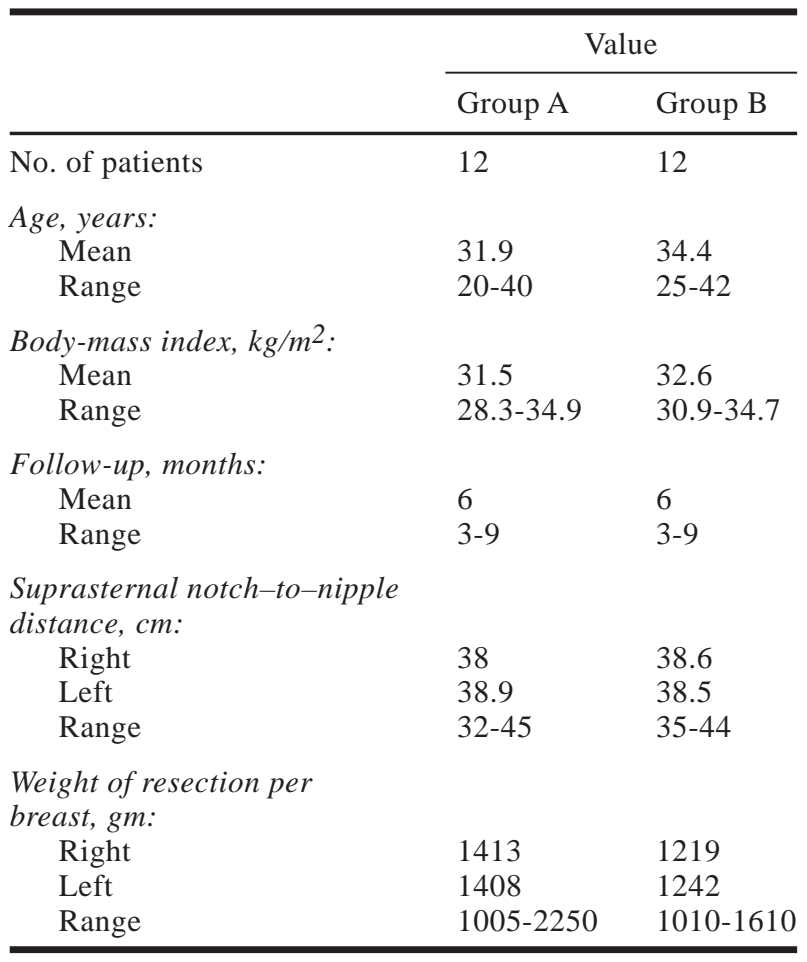

Vessels traveling most superficial to the skin surface, with the largest diameter and linear axis, were marked roughly on the skin surface. For the purpose of documentation, the arterial supply at the entrance to the areola (Fig. 1) was designated as zone 3 . The artery entrance to the breast tissue whether medially, laterally, superiorly or inferiorly was called zone 1 . Finally, zone 2 was designated as the region between these zones (Fig. 2).

\section{Patient markings:}

After determination of the major vessels entering the areola, patients were marked in the standing position. Wise pattern skin excision was planned. Standard sternal notch, midline, inframammary folds, and meridians were marked. The new nipple site was determined at the level of the inframammary fold. The amount of skin excision was determined during breast movement medially and laterally. Keyhole limb angles were determined later. With attention to the preoperatively determined major perforator localizations, pedicles containing the nipple-areola complex were marked roughly. 


\section{Surgical technique:}

All cases were done under general anaesthesia. After standard Wise pattern breast reduction incisions, the pedicle is incised and deepithelialized according to preoperative determination by ultrasonographic color Doppler imaging in group A, and used standard inferior pedicle design for group $\mathrm{B}$. The pedicle containing the areola is raised. The glandular tissue remaining inferior, lateral, or medial to the pedicle is excised en bloc. Whether the areola fits its new position easily is checked. The precise entrance of the vessels to the pedicle enables the surgeon to stay away from the dangerous points at this moment. After rotation and adaptation of the areola, inferior keyhole limbs and T-point sutures are placed. Finally, the skin is closed and drains are placed.

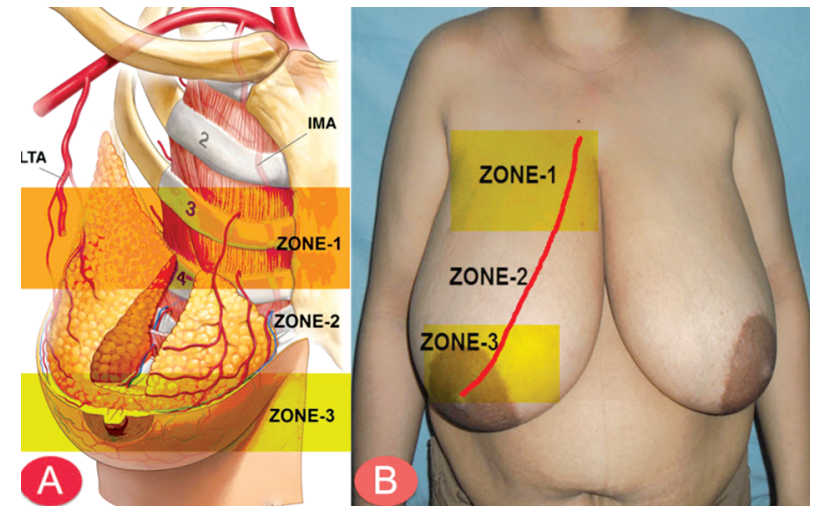

Fig. (1): (A) Illustration showing three zones of NAC vascular supply, (B) Three zones of NAC vascular supply marked on the patient.

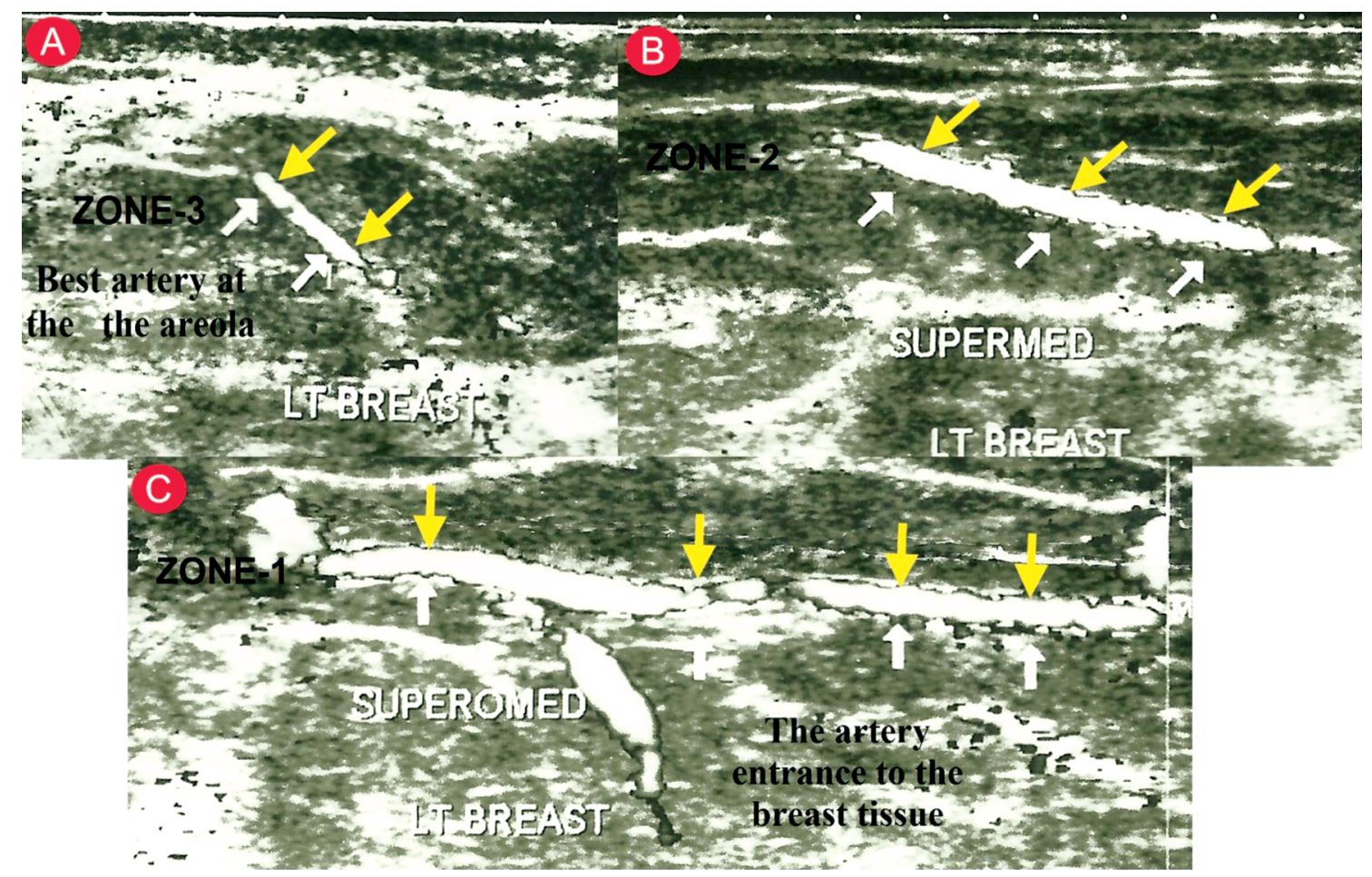

Fig. (2): Doppler images of the main perforators in three different zones. Yellow arrows indicate the artery, (A) Zone 3, (B) Zone 2, (C) Zone 1.

\section{RESULTS}

In group A; after Doppler ultrasonography, Pedicles originating at the internal mammary artery in 10 patients (20 breast) (four cases with superomedial pedicle and Six cases with inferior pedicle), Two cases with superior pedicle (internal mammary artery, second intercostal space), and in group B; all cases underwent reduction mammoplasty using standard inferior pedicle technique.

Mean resection weight was $1413 \mathrm{~g}$ for the right breast and 1408g for the left breast (range, 1005 to $2250 \mathrm{~g}$ ) in group A. In group B; Mean resection weight was $1219 \mathrm{~g}$ for the right breast and $1242 \mathrm{~g}$ for the left breast (range, 1010 to $1610 \mathrm{~g}$ ). None of the patients had areola necrosis. Patients were followed for complications such as minor skin breakdown, seroma, hematoma, infection, and revision requirements. There was no significant difference between Patient Characteristics of the two studied groups preoperatively as regard type of patients, age, weight, and BMI or postoperatively $(p>0.05)$. 


\section{DISCUSSION}

Important preoperative anatomical considerations include the degree and location of breast hypertrophy, the amount of skin excess and its elasticity, and the position of the breast "footprint" on the chest wall. Good results are possible with a variety of techniques and approaches, with surgeon experience being an important factor [4].

In macromastia, large volumes are usually associated with ptosis, axillary extensions, and hollowness of the upper breast pole. However, the vascular supply to the breast remains principally unmodified, although the blood vessels are stretched down, following the hypertrophic and ptotic breast. Because of the increased sternal notch-to-nipple distance, the vascular safety of the nipple-areola complex remains a primary concern. Reduction mammaplasties following the inferior, superior, superomedial or lateral pedicle models provide adequate blood supply to the nipple-areola complex in cases of reduction mammaplasty in "normal-sized" breasts, but might not include sufficient arterial flow to the nipple-areola complex in cases of macromastia [6].

The optimal method of reduction for macromastia patients is not clear. All pedicles for breast reduction in macromastia have some risk of nipple necrosis, even when carefully created. Of the four main arteries available for the nipple, there may be only three dominant vessels, and sometimes the pedicle chosen does not have adequate circulation. Nipple necrosis may be more often a problem with a non-dominant blood supply rather than intraoperative error. Therefor when performing breast reduction surgery, it is very important to preserve the vascularity of tissues as well as sensitivity, especially of the nipple-areolar complex (NAC) [4].
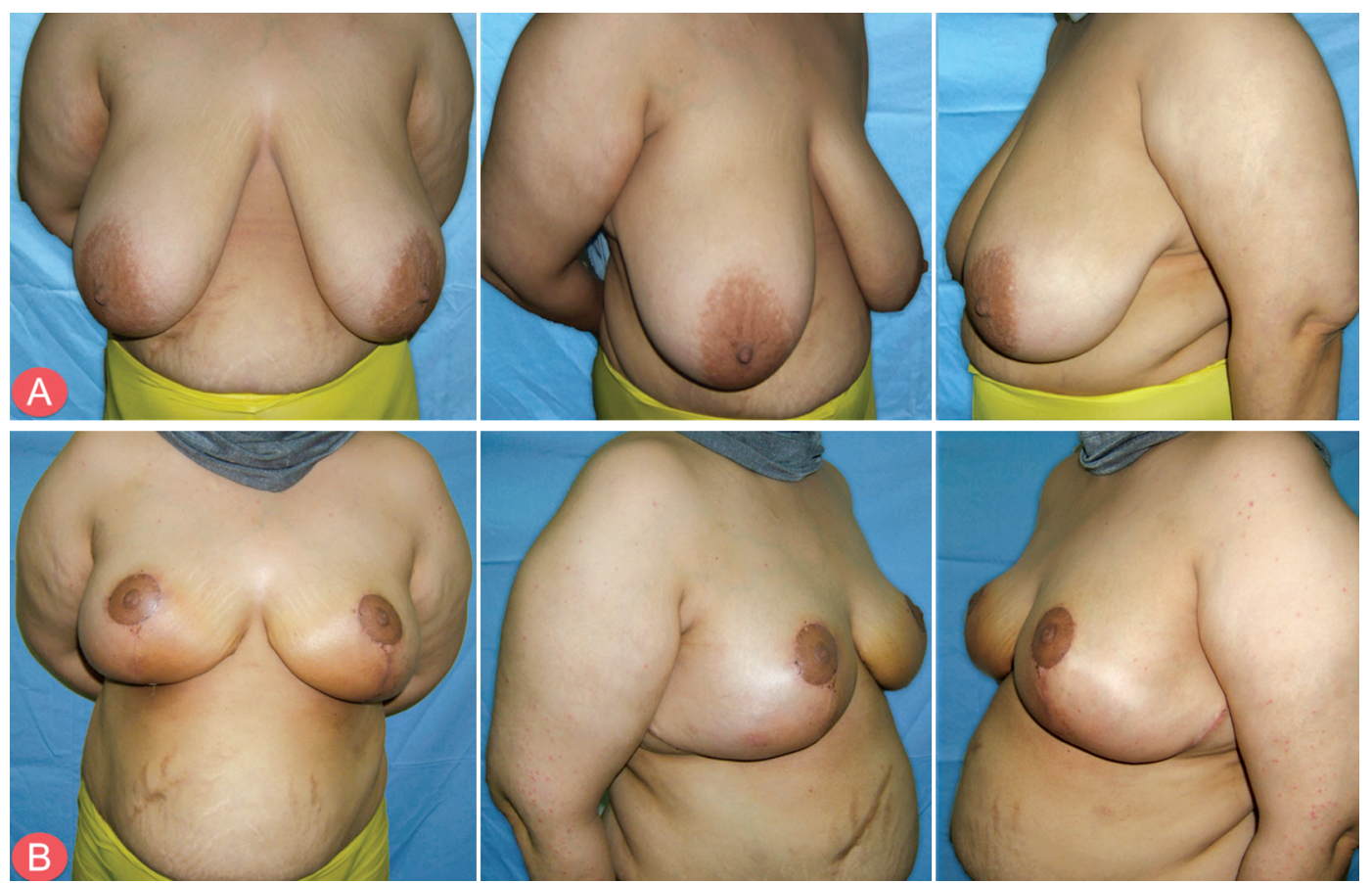

Fig. (3): Photographs of a 35-year-old woman with macromastia (Group A), (A) pre- \& (B) Postoperatively views (frontal, oblique, lateral) (9 months), before resection of $1250 \mathrm{gm}$ from the right and $1200 \mathrm{gm}$ from the left breast, after determination of the pedicel by Preoperative ultrasonographic color Doppler imaging and result in inferior pedicle technique.

The main arteries supplying the nipple-areola complex were reported as internal thoracic, lateral thoracic, thoracoacromial, and intercostal perforators. According to Palmer and Taylor, the internal thoracic artery was the dominant perforator in 70 percent of cases. The only vessel to contribute 100 percent to nipple-areola complex viability [7].

Inferior pedicled breast reduction is the most commonly used method with nipple-areola complex preservation for breasts with large volume and skin envelope. The use of vertical scar mammaplasty is limited for large breasts [8].

Our study had selected two groups of patients, Twenty four (24) female patients (48 breast) both could be suitable candidates for many breast reduction techniques as all our patients had breast macromastia. 
We selected the inferior pedicle technique for group (B) as according to Hammond and Loffredo [9]. The inferior pedicle has been the preferred method of breast reduction for the patients with macromastia, particularly those requiring reductions in excess of $1000 \mathrm{gm}$ per side around the world over the past 40 years, and it has clearly stood the test of time, and it remains the criterion standard against which all other techniques are judged.

We performed a different reduction mammaplasty technique based on Preoperative color Doppler imaging to evaluation of the main nippleareola complex vascular supply for group (A) as according to Basaran et al. [10] that preoperative radiographic evaluation of the main nipple-areola complex vascular supply has certain advantages.

According to Hall-Findlay [11], the breast has two main arterial systems, which are the deep and the superficial systems. The deep system that originates mainly from the fourth intercostal branch of the internal mammary artery constitutes the inferior pedicles. In contrast, the superficial system is composed of the superolateral pedicle (lateral thoracic artery), superior pedicles (internal mammary artery, second intercostal space), and the medial pedicle (internal mammary artery, third intercostal space). These vessels (particularly the superior pedicles) are quite Discussion 121 superficial (approximately $1 \mathrm{~cm}$ deep), which enables them to be found with a color Doppler imaging.

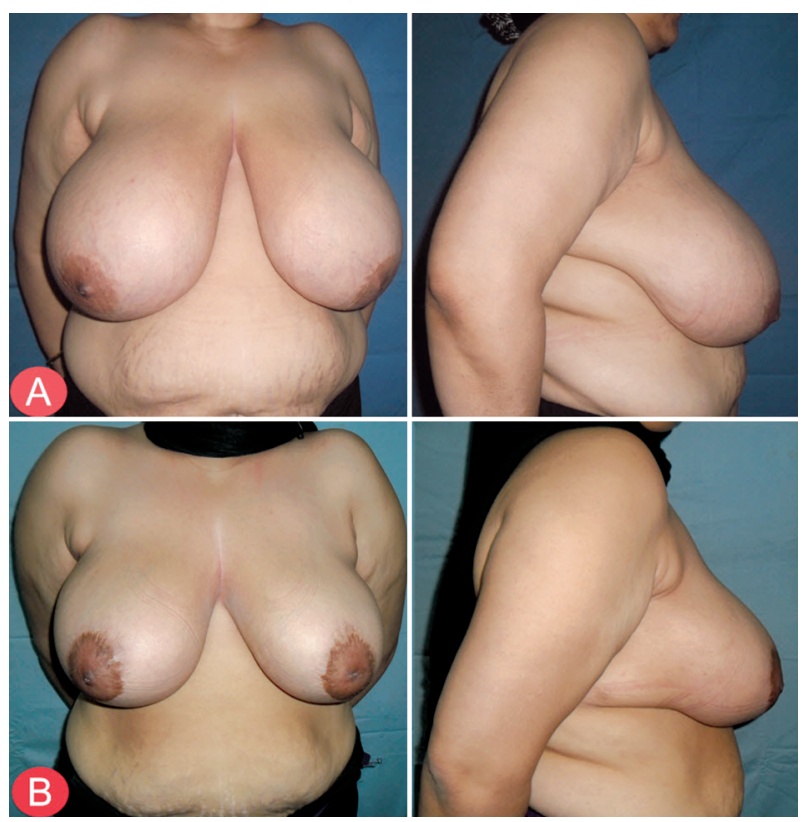

Fig. (4): Photographs of a 38-year-old woman with macromastia (Group A), (A) pre- \& (B) Postoperatively views (frontal, lateral) (12 months), before resection of $1205 \mathrm{gm}$ from the right and $1225 \mathrm{gm}$ from the left breast, after determination of the pedicel by Preoperative ultrasonographic color Doppler imaging and result in superior pedicle technique.
Michelle le Roux et al. [12] noticed that the internal mammary artery perforators and superficial veins were traveling quite superficial around the areola, approximately $1 \mathrm{~cm}$ deep, which was very similar to our findings.

In the literature, breast vasculature assessment before reduction has been used by some authors. For example, Hall-Findlay [11] evaluated 83 patients for the superior pedicle with a Doppler device. She roughly documented the locations of pedicles. However, this evaluation was not performed on macromastia patients, in which the viability of the nipple-areola complex was of great concern.

In our study, we found that the 50\% (6 cases, 12 breasts) of the cases of group (A) relay on deep system that originates mainly from the fourth intercostal branch of the internal mammary artery which constitutes the inferior pedicles. Secondarily $33.3 \%$ ( 4 cases, 8 breasts) of the cases of group (A) relay on superficial system mainly third intercostal space of internal mammary artery which constitutes the superiomedial pedicle. Lastly $16.6 \%$ ( 2 cases, 4 breasts) of the cases of group (A) relay on superficial system mainly second intercostal space of internal mammary artery constitutes the superior pedicles.

Despite Basaran et al. [10], found that main perforators in his study (Sixteen cases) originated mostly from the internal mammary artery and secondarily from the lateral thoracic artery. In our study twelve (12) cases (24 breasts) we found that main perforators originated from the internal mammary artery. Which was very similar to what found in the literature.

In a short letter published by Horta et al. [13], the authors detected the exact location of the superomedial pedicle by a handheld Doppler device. Although the mean reduction weight was $820 \mathrm{~g}$ in their study, they particularly suggested that the use of Doppler would be wise for severe breast hypertrophy cases.

In our study, we decide to use the color Doppler imaging machine (Aplio ${ }^{\mathrm{TM}}$ 400; Toshiba, Japan) Doppler ultrasonography, instead of a handheld Doppler device to be more precise for evaluation of the main nipple-areola complex vascular supply. Which is the same method used by Basaran et al. [10].

With popularization of perforators, preoperative evaluation of these vessels has become more important. The modalities used mostly are magnetic 
resonance or computed tomographic angiography and Doppler ultrasonography [14].

Cina et al. [15] compared multidetector computed tomography and color Doppler ultrasonography for deep inferior epigastric perforator flaps in breast reconstruction. They found that color Doppler imaging was more useful for vessel size. With regard to cost-effectiveness and patient health, Doppler ultrasonography seems to be a better option.

In our study, we visualized the perforators with a sensitive Doppler ultrasonography probe that can detect arteries as small as $0.4 \mathrm{~mm}$ in diameter.

Patients whom undergone coronary artery bypass grafting (CABG), utilizing the internal mammary arteries may compromise blood supply to the breast and lead to breast necrosis during breast reduction surgery [16].

Although we excluded patients with comorbidity as CABG yet we suggests that to doing Preoperative ultrasonographic color Doppler imaging for evaluation of vascular supply of chest wall and breast before performing reduction mammaplasty surgery in patients whom subjected to CABG.

\section{Conclusion:}

Preoperative ultrasonographic color Doppler imaging for evaluation of the main nipple-areola complex vascular supply has certain advantages because patients with macromastia have breasts already distorted anatomically, and it is not wise to rely on standard pedicle designs. Variability of the vessels of each breast even between the same patient the patient, results in risky reduction mammaplasty.

Therefore, we strongly advise using the Preoperative ultrasonographic color Doppler imaging for evaluation and documentation of the main nipple-areola complex vascular supply that specify which type reduction mammaplasty techniques for every patient.

We suggest to add ultrasonographic color Doppler imaging for evaluation of the main nippleareola complex vascular supply as modalities of preoperative radiological imaging of the breast with breast ultrasound and breast mammogram.

Secondary cases with macromastia after primary reduction mammaplasty can be a challenging problem. It is important to know the limitations and risks of repeated breast reduction especially in whom the NAC needs to be transposed.
We suggest using the Preoperative ultrasonographic color Doppler imaging for evaluation of vascular supply for Secondary cases of reduction mammaplasty especially with unknown primary technique.

We also suggest that doing Preoperative ultrasonographic color Doppler imaging for evaluation of vascular supply of chest wall and breast after using internal thoracic arteries for cardiac revascularization before performing CABG surgery, and when performing reduction mammaplasty surgery in patients whom subjected to CABG to prevent potential ischemic flap necrosis and its subsequent complications.

The Inferior pedicle technique is easy to learn and safe in all reduction mammaplasty scenarios, regardless of the size of the breast.

\section{REFERENCES}

1- Rohrich R.J., Gosman A.A., Brown S.A., et al.: Current preferences for breast reduction techniques: A survey of board-certified plastic surgeons 2002. Plast. Reconstr. Surg., 114: 1724-33, 2004.

2- Breiting L.B., Henriksen T.F., Kalialis A.V., et al.: A prospective study of short and long-term cosmetic outcome after reduction mammaplasty from three different perspectives: The patient, a department surgeon, and an independent private practitioner in plastic surgery. Plast. Reconstr. Surg., 130: 273-281, 2012.

3- Sterodimas A., Pineda E.F., V Meirelles, et al.: Maximizing nipple graft survival after performing free nipple-areolar complex reduction mammaplasty. J. Plast. Reconstr. Aesthet. Surg., 61: 971-972, 2008.

4- Hall-Findlay E.J. and Shestak K.C.: Breast Reduction, Plast. Reconstr. Surg., 136 (4): 531e-44e, 2015.

5- Van Deventer P.V., Page B.J. and Graewe F.R.: The safety of pedicles in breast reduction and mastopexy procedures. Aesthetic Plast. Surg., 32 (2): 307-12, 2008.

6- Mojallal A1, Moutran M., Shipkov C., et al.: Breast reduction in gigantomastia using the posterosuperior pedicle: An alternative technique, based on preservation of the anterior intercostal artery perforators. Plast. Reconstr. Surg. Jan., 125 (1): 32-43, 2010.

7- Palmer J.H. and Taylor G.I.: The vascular territories of the anterior chest wall. Br. J. Plast. Surg., 39: 287-299, 1986.

8- McCulley S.J. and Schaverien M.V.: Superior and superomedial pedicle wise-pattern reduction mammaplasty: Maximizing cosmesis and minimizing complications. Ann. Plast. Surg., 63: 128-134, 2009.

9- Hammond D.C. and Loffredo M.: Breast reduction. Plast. Reconstr. Surg., 129 (5): 829e-839e, 2012.

10- Başaran K., Ucar A., Guven E., et al.: Ultrasonographically determined pedicled breast reduction in severe gigantomastia. Plast. Reconstr. Surg., 128 (4): 252e-259e, 2011. 
11- Hall-Findlay E.J.: Applied anatomy: Key concepts for modern breast surgery. In: Aesthetic Breast Surgery: Concepts and Techniques. St. Louis: Quality Medical, 67-98, 2011.

12- Michelle le Roux C., Kiil B.J., Pan W.R., et al.: Preserving the neurovascular supply in the Hall-Findlay superomedial pedicle breast reduction: An anatomical study. J. Plast. Reconstr. Aesthet. Surg., 63 (4): 655-62, 2010.

13- Horta R., Silva P., Filipe R., et al.: Use of Doppler in breast reduction with superomedial or superolateral pedicles. Aesthetic Plast. Surg., 34: 680-681, 2010.
14- Newman T.M., Vasile J., Levine J.L., et al.: Perforator flap magnetic resonance angiography for reconstructive breast surgery: A review of 25 deep inferior epigastric and gluteal perforator artery flap patients. J. Magn Reson Imaging, 31: 1176-1184, 2010.

15- Cina A., Salgarello M., Barone-Adesi L., et al.: Planning breast reconstruction with deep inferior epigastric artery perforating vessels: Multidetector CT angiography versus color Doppler US. Radiology, 255: 979-987, 2010.

16- Rashid A., Haj Basheer M. and Khan K.: Breast necrosis following harvest of internal mammary artery. Br. J. Plast. Surg., 57 (4): 366-368, 2004. 